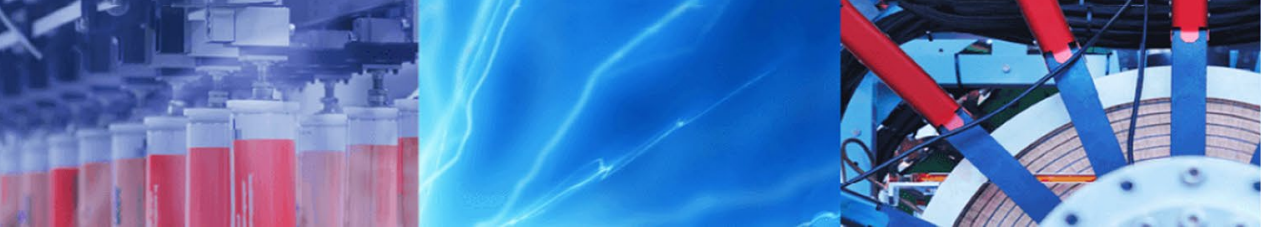

Research Article

\title{
Influence of viscous dissipation and double stratification on MHD Oldroyd-B fluid over a stretching sheet with uniform heat source
}

\author{
K. Kalyani ${ }^{1} \cdot$ N. Seshagiri Rao ${ }^{2}$ O O. D. Makinde ${ }^{3}$ M. Gnaneswara Reddy ${ }^{4}$ M. V. V. N. L. Sudha Rani ${ }^{4}$
}

(c) Springer Nature Switzerland AG 2019

\begin{abstract}
The present report explores the discussion on 3D flow of dual stratified Oldroyd-B fluid in the direction of thermal radiation, viscous dissipation and heat source/sink. Mathematical modeling is formulated with an applied magnetic field through a stretching surface. The present flow governing system has been transformed as nonlinear ODE via suitable transformations and then concluded by using bvp4c. The graphs are described and illustrated for various non-dimensional parameters. Thermal stratification and Prandtl number parameters reduce the temperature, whereas thermal radiation and heat source parameters show reverse behavior. Numerical results are used to obtain the values of frictional drag, rate of heat and mass transfers. Finally, numerical results are compared with previous established work in a limiting case.
\end{abstract}

Keywords 3D Oldroyd-B fluid · Dual stratification $\cdot M H D \cdot T h e r m a l$ radiation $\cdot$ Heat source/sink

\section{Introduction}

In present days, the review of non-Newtonian fluids is an interesting concept to the novel investigators in an aspect of their utilizations in the production and technology. Foodstuffs, material industries, shampoos, form oil and bioengineering are some examples of non-Newtonian fluids. Fluids those does not pursue the Newton's law are called non-Newtonian fluids. Maxwell [1] developed the one-dimensional Maxwell fluid model, which includes a subclass of rate-type fluids, and it is found to have only relaxation time but not retardation time. Oldroyd [2] introduced the Oldroyd-B fluid; it is the branch of nonNewtonian fluid. It shows both relaxation and retardation time properties of a non-Newtonian fluid. Fetecau [3] investigated the Maxwell fluid is highly applicable for large relaxation time polymer extrusion process, drawing of metals. Temperature and concentration effects on
non-Newtonian fluid were investigated by Chuo-Jeng Huang [4]. Abdul Gaffar et al. [5] were analyzed by a nonNewtonian fluid across a non-isothermal wedge, utilizing implicit finite difference method for obtaining graphical representations. Soret and Dufour effects on Casson and Maxwell fluids across a stretching sheet were investigated by Ramana Reddy et al. [6] by implementing RK Felhberg technique for obtaining solutions and presenting graphical representations to investigate the consequences for both the non-Newtonian fluids. Raju et al. [7] analyzed the impacts of non-Newtonian fluid over homogeneous/ heterogeneous reactions, exhibiting graphical representations for both stretching and shrinking surfaces. Consequently, many researchers were involved in examining the different physical conditions of Oldroyd-B fluid in various regions (see [8-17]).

Magnetohydrodynamic flow across a stretching/ shrinking disk was analyzed by Khuzaimah Soid et al. [18],

K. Kalyani, kalyani.namana@gmail.com; N. Seshagiri Rao, seshu.namana@gmail.com; O. D. Makinde, makinded@gmail.com; M. Gnaneswara Reddy, mgrmaths@gmail.com; M. V.V. N. L. Sudha Rani, sudharani0310@gmail.com | 1 Department of Science and Humanities, Vignan's Foundation for Science, Technology and Research, Vadlamudi, Andhra Pradesh 522213, India. ${ }^{2}$ Department of Applied Mathematics, School of Applied Natural Science, Adama Science and Technology University, Post Box No. 1888, Adama, Ethiopia. ${ }^{3}$ Faculty of Military Science, Stellenbosch University, Stellenbosch, South Africa. ${ }^{4}$ Department of Mathematics, Acharya Nagarjuna University, Ongole Campus, Ongole, Andhra Pradesh, India. 
investigating stability of the multiple solutions. Hydromagnetic flow over a stretchable curved space was studied by Hayat et al. [19] and presented graphical representations for friction factor, rate of heat and mass transfers to estimate curvature. MHD flow of a nano-fluid through a saturating permeability porous was analyzed by Siva Kumar Reddy et al. [20] by using HAM and carried out the study of numerical representations. HAM solutions on MHD 3D effects were scrutinized by Hayat et al. [21] and concluded that temperature and concentration fields are enhancing for escalating values of $M$. Influence of heat transfer effects on MHD flow of thermal radiation and viscous dissipation was investigated by Nayak [22]. Ganesh Kumar et al. [23] distinguish the Joule heating and viscous dissipation on 3D Oldroyd-B nano-fluid flow along thermal radiation. Impacts of Joule and viscous heating effects on 3D hydromagnetic flow including mass and heat fluxes were examined by Muhammad et al. [24]. Jaber [25] investigated the power law heat flux with Joule heating and viscous dissipation on 3D hydromagnetic through a porous stretching sheet.

Heat and mass transfer flows across a stretching sheet with double stratification have a main role in polymer production, chemical industries, wire drawing, paper production etc. Heat and mass transfer has several applications like glass fiber, extrusion process and damage of crops due to freezing. Impact of hydromagnetic tangent-hyperbolic nano-fluid-was explored by Sajid et al. [26] and analytically solved by using HAM. Impact of heat and mass transfer effects across a stretching sheet was considered by Mishra and Bhatti [27] determined numerically with the aid of successive linearization method and Chebyshev spectral collocation method. 2D heat and mass transfer effects of Casson fluid in the presence of wall mass transfer was explained by Ali et al. [28]. 2D boundary layer flow of double stratification of Eyring-Powell fluid was probed by Khalil et al. [29] and plotted graphical representations for friction factor, rate of heat and mass transfers for both the cylinder and plate. Hydromagnetic non-Newtonian fluid across a stretching cylinder with double stratification was examined by Khalil et al. [30] and concluded that $\varepsilon_{1}$ enhances the rate of heat transfer decays.

In the raised references, the influence of viscous dissipation is not addressed. The objective of the current paper carried out the study of 3D flow of Oldroyd-B dual stratified fluid through a stretching sheet in direction of temperaturedependent heat source/sink, chemical reaction and viscous dissipation in the magnetic field.

\section{Model problem}

We consider an incompressible steady 3D flow of an Oldroyd-B fluid past a stretching surface at $z=0$. The physical interpretation of the flow is presented in Fig. 1. The fluid flow is taken at constant magnetic field $B_{0}$ applied in the normal direction. The magnetic Reynold's number is given to be small, and thus, the induced magnetic field is negligible. Let $u_{w}=m x$ and $v_{w}=n y$ be the velocities along $x$ and $y$ directions of the stretching surface. Thermal and solutal stratifications are taken into account in the direction of viscous dissipation, thermal radiation, temperature-dependent heat source/sink and chemical reaction parameter.

The constitutive equation for 3D Oldroyd-B fluid can be addressed as [31] at pages 221-223.

$\tau=-\mathrm{PI}+\mathrm{S}$

$S+\lambda_{1} \frac{\delta}{\delta t} S=\mu\left(A_{1}+\lambda_{2} \frac{\delta A_{1}}{\delta t}\right)$

where $\lambda_{1}$ and $\lambda_{2}$ are the relaxation time and retardation time, respectively, and $\frac{\delta}{\delta t}$ is the covariant differentiation. The first Rivlin-Ericksen tensor $A_{1}$ is

$A_{1}=\operatorname{grad} U+(\operatorname{grad} U)^{*}$

where *denotes the transpose of the matrix and the velocity $U$ is taken as

$U=\left(u_{1}(x, y, z), u_{2}(x, y, z), u_{3}(x, y, z)\right)$

The definition of $\frac{\delta}{\delta t}$ is

$\frac{\delta a_{i}}{\delta t}=\frac{\partial a_{i}}{\partial t}+u_{r} a_{i, r}-u_{i, r} a_{r}$

The governing equations of the 3D Oldroyd-B fluid flow under the boundary layer assumptions are [32, 33]:

$\frac{\partial u}{\partial x}+\frac{\partial v}{\partial y}+\frac{\partial w}{\partial z}=0$

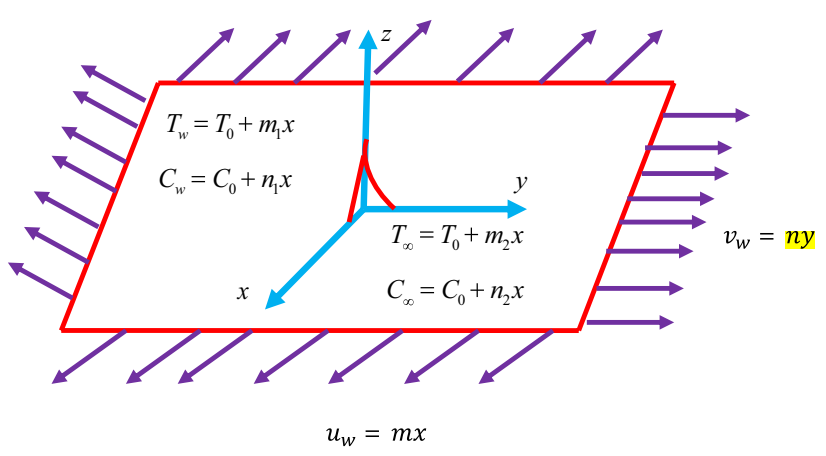

Fig. 1 Physical model and coordinate system 


$$
\begin{aligned}
& u \frac{\partial u}{\partial x}+v \frac{\partial u}{\partial y}+w \frac{\partial u}{\partial z}+\lambda_{1}\left(u^{2} \frac{\partial^{2} u}{\partial x^{2}}+v^{2} \frac{\partial^{2} u}{\partial y^{2}}+w^{2} \frac{\partial^{2} u}{\partial z^{2}}+2 u v \frac{\partial^{2} u}{\partial x \partial y}+2 u w \frac{\partial^{2} u}{\partial x \partial z}+2 v w \frac{\partial^{2} u}{\partial y \partial z}\right) \\
& =v\left(\frac{\partial^{2} u}{\partial z^{2}}+\lambda_{2}\left(u \frac{\partial^{3} u}{\partial x \partial z^{2}}+v \frac{\partial^{3} u}{\partial y \partial z^{2}}+w \frac{\partial^{3} u}{\partial z^{3}}-\frac{\partial u}{\partial x} \frac{\partial^{2} u}{\partial z^{2}}-\frac{\partial u}{\partial y} \frac{\partial^{2} u}{\partial z^{2}}-\frac{\partial u}{\partial z} \frac{\partial^{2} u}{\partial z^{2}}\right)\right)-\frac{\sigma B_{0}^{2} u}{\rho} \\
& u \frac{\partial v}{\partial x}+v \frac{\partial v}{\partial y}+w \frac{\partial v}{\partial z}+\lambda_{1}\left(u^{2} \frac{\partial^{2} v}{\partial x^{2}}+v^{2} \frac{\partial^{2} v}{\partial y^{2}}+w^{2} \frac{\partial^{2} v}{\partial z^{2}}+2 u v \frac{\partial^{2} v}{\partial x \partial y}+2 u w \frac{\partial^{2} v}{\partial x \partial z}+2 v w \frac{\partial^{2} v}{\partial y \partial z}\right) \\
& =v\left(\frac{\partial^{2} v}{\partial z^{2}}+\lambda_{2}\left(u \frac{\partial^{3} v}{\partial x \partial z^{2}}+v \frac{\partial^{3} v}{\partial y \partial z^{2}}+w \frac{\partial^{3} v}{\partial z^{3}}-\frac{\partial v}{\partial x} \frac{\partial^{2} v}{\partial z^{2}}-\frac{\partial v}{\partial y} \frac{\partial^{2} v}{\partial z^{2}}-\frac{\partial v}{\partial z} \frac{\partial^{2} v}{\partial z^{2}}\right)\right)-\frac{\sigma B_{0}^{2} v}{\rho} \\
& u \frac{\partial T}{\partial x}+v \frac{\partial T}{\partial y}+w \frac{\partial T}{\partial z}=\frac{k}{\rho c_{p}} \frac{\partial^{2} T}{\partial z^{2}}+\frac{16 \sigma^{*} T_{\infty}^{3}}{3 \rho c_{p} k^{*}} \frac{\partial^{2} T}{\partial z^{2}}+\frac{\mu}{\rho c_{p}}\left[\left(\frac{\partial u}{\partial z}\right)^{2}+\left(\frac{\partial v}{\partial z}\right)^{2}\right] \\
& +\frac{Q_{0}}{\rho c_{p}}(T-T \infty)+\frac{\sigma B_{0}^{2}}{\rho}\left(u^{2}+v^{2}\right)
\end{aligned}
$$

$u \frac{C}{x}+v \frac{C}{y}+w \frac{C}{z}=D \frac{\partial^{2} C}{z^{2}}-k_{0}\left(C-C_{\infty}\right)$

where $u, v$ and $w$ represent the velocity components in $3 \mathrm{D}$, respectively, $\lambda_{1}$ and $\lambda_{2}$ are the times of relaxation and retardation, $T$ and $C$ are the fluid temperature and concentration, $v, \sigma, \rho$ and $\mu$ denote the kinematic viscosity, thermal diffusivity, density and dynamic viscosity of the fluid, $k$ the medium thermal conductivity, $k^{*}$ the absorption coefficient, $\sigma^{*}$ the Stefen-Boltzman constant, $Q_{0}$ the heat generation $\left(Q_{0}>0\right)$ or absorption $\left(Q_{0}<0\right)$ coefficient, $c_{p}$ the specific heat at constant pressure, $D$ the fluid mass diffusivity and $k_{0}$ the chemical reaction. The applicable boundary conditions to the present flow are:

$u=U_{w}, v=V_{w}, w=0, T=T_{w}=T_{0}+m_{1} x$,

$C=C_{w}=C_{0}+n_{1} x \quad$ at $z=0$

$u, v \rightarrow 0, T \rightarrow T_{\infty}=T_{0}+m_{2} x, C \rightarrow C_{\infty}=C_{0}+n_{2} x, \quad$ as $z \rightarrow \infty$

where $m_{1}, m_{2}, n_{1}, n_{2}$ are dimensional constants, $T_{w}$ and $T_{\infty}$ are uniform temperature and temperature of the fluid in the free stream with $T_{w}>T_{\infty}, C_{w}$ and $C_{\infty}$ are the uniform concentration and concentration of the fluid in the free stream with $C_{w}>C_{\infty}$ and the reference temperature, reference concentration are denoted by $T_{0}, C_{0}$, respectively.

\section{Method of solution}

The recommended similarity variables are given by: $f^{\prime}(\eta)=\frac{u}{m x}, \quad g^{\prime}(\eta)=\frac{v}{n y}, \quad f(\eta)+g(\eta)=-\frac{w}{\sqrt{m v}}$

$\eta=z \sqrt{\frac{m}{v}}, \quad \theta(\eta)=\frac{T-T_{\infty}}{T_{w}-T_{0}}, \quad \phi(\eta)=\frac{C-C_{\infty}}{C_{w}-C_{0}}$

Equation (6) convinced automatically by using Eq. (13) and Eqs. (7)-(12)

$$
\begin{aligned}
& f^{\prime \prime \prime}+(f+g) f^{\prime \prime}-f^{\prime 2}+\beta_{1}\left(2(f+g) f^{\prime} f^{\prime}-(f+g)^{2} f^{\prime \prime \prime}\right) \\
& \quad+\beta_{2}\left(\left(f^{\prime \prime}+g^{\prime \prime}\right) f^{\prime \prime}-(f+g) f^{i v}\right)-M f^{\prime}=0 \\
& g^{\prime \prime \prime}+(f+g) g^{\prime \prime}-g^{\prime 2}+\beta_{1}\left(2(f+g) g^{\prime} g^{\prime \prime}-(f+g)^{2} g^{\prime \prime \prime}\right) \\
& \quad+\beta_{2}\left(\left(f^{\prime \prime}+g^{\prime \prime}\right) g^{\prime \prime}-(f+g) g^{i v}\right)-M g^{\prime}=0 \\
& \left(1+\frac{4}{3} N r\right) \theta^{\prime \prime}-p r\left(f^{\prime} \theta+f^{\prime} \varepsilon-(f+g) \theta^{\prime}\right. \\
& \left.\quad-\left(E c_{x} f^{\prime \prime 2}+E c_{y} g^{\prime \prime 2}\right)-Q \theta-M\left(E c_{x} f^{\prime 2}+E c_{y} g^{\prime^{2}}\right)\right)=0
\end{aligned}
$$

$\phi^{\prime \prime}+S c\left((f+g) \phi^{\prime}-f^{\prime} \phi-\varepsilon_{2} f^{\prime}-\gamma \phi\right)=0$

$f^{\prime}(0)=1, g^{\prime}(0)=c, f(0)=0, g(0)=0, \theta(0)=1-\varepsilon_{1}, \phi(0)=1-\varepsilon_{2}$

$f^{\prime}(\infty) \rightarrow 0, g^{\prime}(\infty) \rightarrow 0, \theta(\infty) \rightarrow 0, \phi(\infty) \rightarrow 0$

where superscript expresses the differentiation with respect to $\eta$. 
$M\left(=\frac{\sigma B_{0}^{2}}{\rho m}\right)$ the magnetic field parameter, $c\left(=\frac{n}{m}\right)$ the stretching ratio parameter, $\beta_{1}\left(=\lambda_{1} m\right)$ and $\left.\beta_{2}\left(=\lambda_{2} n\right)\right)$ are Deborah numbers, $\operatorname{Pr}\left(=\frac{\rho c_{p} v}{k}\right)$ the Prandtl number, $\operatorname{Nr}\left(=\frac{4 \sigma^{*} T_{\infty}^{3}}{k k^{*} u^{2}}\right)$ the thermal radiation parameter, $E c_{x}=\frac{k k^{*} u_{w}^{2}}{c_{p}\left(T_{w}-T_{0}\right)}, E c_{y}=\frac{v_{w}^{2}}{c_{p}\left(T_{w}-T_{\infty}\right)}$ the Eckert number along $x$ and $y$ directions, $Q\left(=\frac{Q_{0}}{\rho c_{p} m}\right)$ the heat source/sink parameter, $\varepsilon_{1}\left(=\frac{m_{2}}{m_{1}}\right)$ the thermal stratification parameter, $\varepsilon_{2}\left(=\frac{n_{2}}{n_{1}}\right)$ the solutal stratification parameter, $\operatorname{Sc}\left(=\frac{v}{D}\right)$ the Schmidt number, $\gamma\left(=k_{0} / m\right)$ the chemical reaction parameter.

In Eqs. (14) and (15), if

1. $\beta_{2}=0$, then the present problem performs Maxwell fluid model.

2. $\beta_{1}=\beta_{2}=0$, then the present problem performs classical Newtonian fluid.

The local frictional drag coefficients, heat and mass transfer rates are

$$
\begin{aligned}
& C_{f_{x z}} \sqrt{R e_{x}}=\frac{1+\beta_{1}}{1+\beta_{2}} f^{\prime \prime}(0) \\
& C_{f_{y z}} \sqrt{R e_{y}}=\frac{1+\beta_{1}}{1+\beta_{2}} g^{\prime \prime}(0) \\
& N u_{x} / R e_{x}^{1 / 2}=-\left(1+\frac{4}{3} N r\right) \theta^{\prime}(0)
\end{aligned}
$$

$S h_{x} / R e_{x}^{1 / 2}=-\phi^{\prime}(0)$

Table 1 Comparison of local skin friction coefficient $f^{\prime \prime}(0)$ with Ibrahim et al. [34] and Hayat et al. [35] when $\beta_{1}=\beta_{2}=\operatorname{Pr}=N r=E c_{x}=E c_{y}=Q=S c=\gamma=\varepsilon_{1}=\varepsilon_{2}=0 \quad$ for Newtonian fluid

\begin{tabular}{llll}
\hline$M$ & Ibrahim et al. [34] & Hayat et al. [35] & $f^{\prime \prime}(0)$ \\
\hline 0.0 & -1.2808 & -1.2808 & -1.2808 \\
1.0 & -1.4142 & -1.4142 & -1.4142 \\
5.0 & -2.4494 & -2.4494 & -2.4494 \\
\hline
\end{tabular}

where $\operatorname{Re}_{x}\left(=\frac{u_{w} x}{v}\right)$ is the local Reynolds number.

The highly coupled nonlinear differential Eqs. (14)-(17) that are in subject to the corresponding boundary conditions (18)-(19) are worked out by employing bvp4c and obtained numerical values. The ability of numerical method is approved by examining the results, that is $f^{\prime \prime}(0)$ of the present analysis with those calculated by Ibrahim et al. [31], Hayat et al. [32] in the limiting case for a Newtonian fluid when $\operatorname{Pr}=N r=E c_{x}=E c_{y}=Q=S c=\gamma=\varepsilon_{1}=\varepsilon_{2}=\beta_{1}=\beta_{2}=0$ Table 1 explains that the outcomes are in wonderful agreement (Tables 2, 3).

\section{Results and discussion}

The investigation is focused to explore the consequences of double stratification on 3D flow of an Oldroyd-B fluid past a stretching surface under the effect of a transverse magnetic field, thermal radiation in the direction of temperature-dependent heat source/sink, viscous heating and first-order chemical reaction. To understand the physics of the problem under investigation, the flow variables, viz. velocity, temperature and species concentration, are computationally evaluated at some fixed values of nondimensional parameters $\beta_{1}=\beta_{2}=0.5, \varepsilon_{1}=\varepsilon_{2}=0.1$, $M=0.5, \operatorname{Pr}=1.2, E c_{x}=E c_{y}=0.1, S c=0.8, \gamma=0.1, Q=0.2$, $\mathrm{Nr}=1.0, c=0.8$ except the diverse values as addressed in the respective figures and illustrated through plots. Physical interpretations of the results are discussed in detail.

Figures 2, 3 and 4 indicate the velocity, temperature and concentration plots for various values of Deborah number $\left(\beta_{1}\right)$. Figure 2 shows that velocity decreases with increasing $\beta_{1}$ as it reduces the drag experienced by the stretching surface. Temperature distribution is seen to increase in the thermal boundary layer as $\beta_{1}$ enlarges and the associated thermal boundary layer thickness increases due to the reduction in velocity in the boundary layer. Species concentration qualitatively follows the same behavior as that of temperature.
Table 2 Comparison of various values of $c$ with Hayat et al.

\begin{tabular}{|c|c|c|c|c|c|c|c|c|}
\hline \multirow[t]{2}{*}{ C } & \multicolumn{2}{|c|}{ Hayat et al. [36] } & \multicolumn{2}{|c|}{$\begin{array}{l}\text { Rudraswamy et al. } \\
\text { [37] }\end{array}$} & \multicolumn{2}{|c|}{ Ganesh et al. [38] } & \multicolumn{2}{|c|}{ Present results } \\
\hline & $-f^{\prime \prime}(0)$ & $-g^{\prime \prime}(0)$ & $-f^{\prime \prime}(0)$ & $-g^{\prime \prime}(0)$ & $-f^{\prime \prime}(0)$ & $-g^{\prime \prime}(0)$ & $-f^{\prime \prime}(0)$ & $-g^{\prime \prime}(0)$ \\
\hline 0 & 1 & 0 & 1 & 0 & 1 & 0 & 1 & 0 \\
\hline 0.25 & 1.048810 & 0.19457 & 1.04881 & 0.19457 & 1.04881 & 0.19457 & 1.04882 & 0.19458 \\
\hline 0.5 & 1.093095 & 0.465205 & 1.09309 & 0.46522 & 1.093095 & 0.46522 & 1.093095 & 0.46521 \\
\hline 0.75 & 1.134500 & 0.794620 & 1.13450 & 0.79462 & 1.13450 & 0.79462 & 1.13449 & 0.79464 \\
\hline 1.0 & 1.173721 & 1.173721 & 1.17372 & 1.17372 & 1.17372 & 1.17372 & 1.17373 & 1.17373 \\
\hline
\end{tabular}
[36], Rudraswamy et al. [37] and Ganesh et al. [38] 
Table 3 Numerical values of $-\theta^{\prime}(0)$ and $-\phi^{\prime}(0)$ for some values of $\operatorname{Pr}=1.2, E C=0.1$, $S C=0.8, \gamma=0.1, Q=0.2$ and $\mathrm{Nr}=1.0$

\begin{tabular}{|c|c|c|c|c|c|c|}
\hline$\beta_{1}$ & $\beta_{2}$ & $M$ & $\varepsilon_{1}$ & $\varepsilon_{2}$ & $-\theta^{\prime}(0)$ & $-\phi^{\prime}(0)$ \\
\hline 0.2 & 0.5 & 0.5 & 0.1 & 0.1 & 0.602263 & 1.025792 \\
\hline 0.7 & & & & & 0.499512 & 0.964644 \\
\hline 1.2 & & & & & 0.413240 & 0.914331 \\
\hline 1.7 & & & & & 0.341643 & 0.872434 \\
\hline \multirow[t]{4}{*}{0.5} & 0.5 & 0.5 & 0.1 & 0.1 & 0.546207 & 0.987944 \\
\hline & 0.7 & & & & 0.586368 & 1.013971 \\
\hline & 1.0 & & & & 0.631435 & 1.043420 \\
\hline & 1.5 & & & & 0.682537 & 1.077392 \\
\hline \multirow[t]{4}{*}{0.5} & 0.5 & 1.0 & 0.1 & 0.1 & 0.532954 & 0.969914 \\
\hline & & 2.0 & & & 0.487148 & 0.936630 \\
\hline & & 4.0 & & & 0.412381 & 0.882465 \\
\hline & & 6.0 & & & 0.353748 & 0.840044 \\
\hline \multirow[t]{4}{*}{0.5} & 0.5 & 0.5 & 0.1 & 0.1 & 0.546207 & 0.987944 \\
\hline & & & 0.2 & & 0.513247 & 0.987944 \\
\hline & & & 0.3 & & 0.480288 & 0.987944 \\
\hline & & & 0.4 & & 0.447328 & 0.987944 \\
\hline \multirow[t]{4}{*}{0.5} & 0.5 & 0.5 & 0.1 & 0.0 & 0.578881 & 1.056408 \\
\hline & & & & 0.1 & 0.578881 & 0.990429 \\
\hline & & & & 0.2 & 0.578881 & 0.924451 \\
\hline & & & & 0.3 & 0.578881 & 0.858473 \\
\hline
\end{tabular}

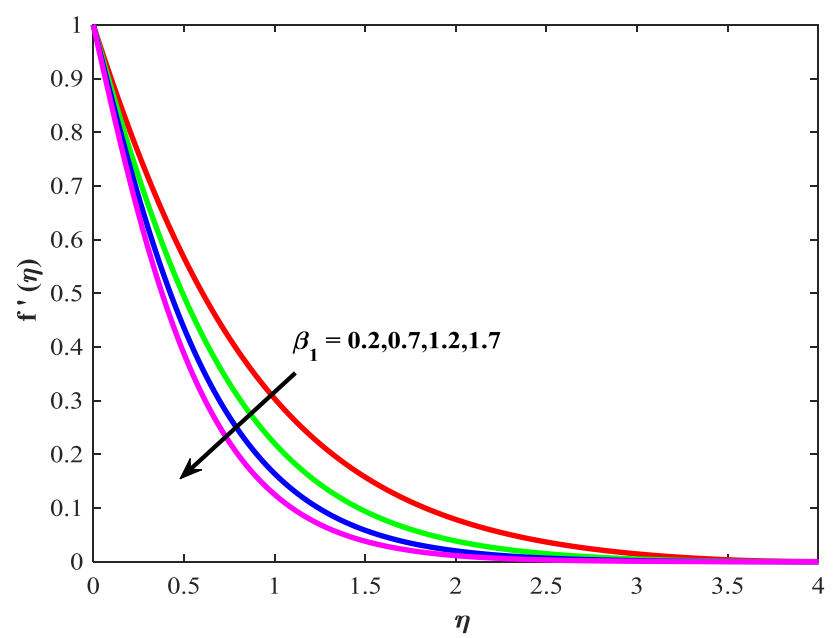

Fig. 2 Deviation of $\beta_{1}$ on $f^{\prime}(\eta)$

Figures 5, 6 and 7 depict the deviations in velocity, temperature and concentration profiles for distinct values of $\beta_{2}$, and it is observed that the velocity increases with increasing $\beta_{2}$ as it enhances the drag experienced by stretching plane. The profile of temperature and thermal boundary layer thickness is reducing objectives of $\beta_{2}$. The Deborah numbers $\beta_{1}$ and $\beta_{2}$ have reversal effects on the profile of temperature in Figs. 3 and 6 . Higher relaxation time inspires to larger temperature, while larger retardation time leads to lower temperature. Therefore, an increment in $\beta_{1}$ produces a high temperature while higher $\beta_{2}$

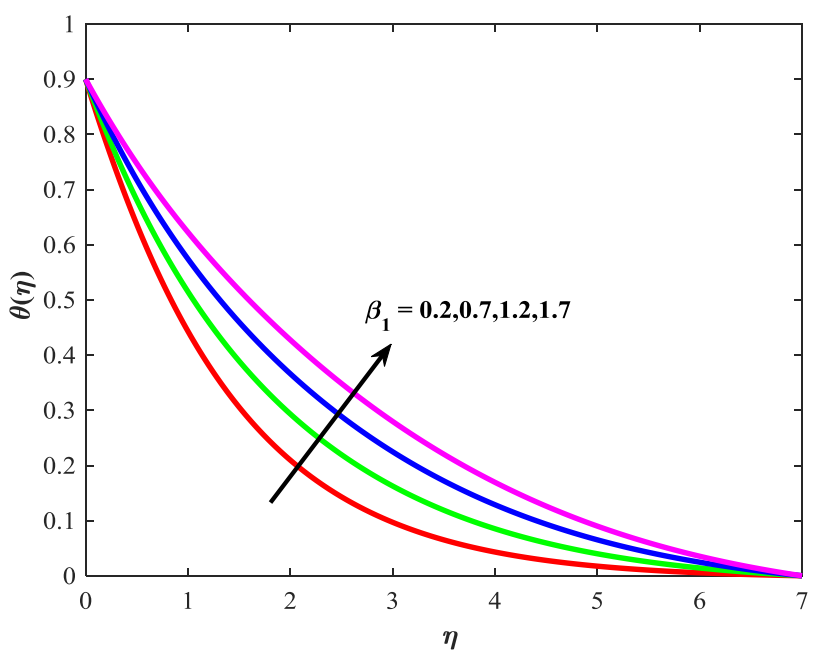

Fig. 3 Deviation of $\beta_{1}$ on $\theta(\eta)$

illustrates the lower temperature. Concentration profile follows the similar behavior as that of temperature.

Figure 8 presents the consequences of the parameter $M$ on the profile of velocity. It is clear that enhancing the strength of $M$ resulted in diminishing the velocity near the plate $(0 \leq \eta \leq 3)$ and outside this range its influence is negligible. Velocity is observed to decrease with the increase in $M$ accompanied by thinning of boundary layers. This reduction can be attributed to the fact that the transverse magnetic field generates the Lorentz force 

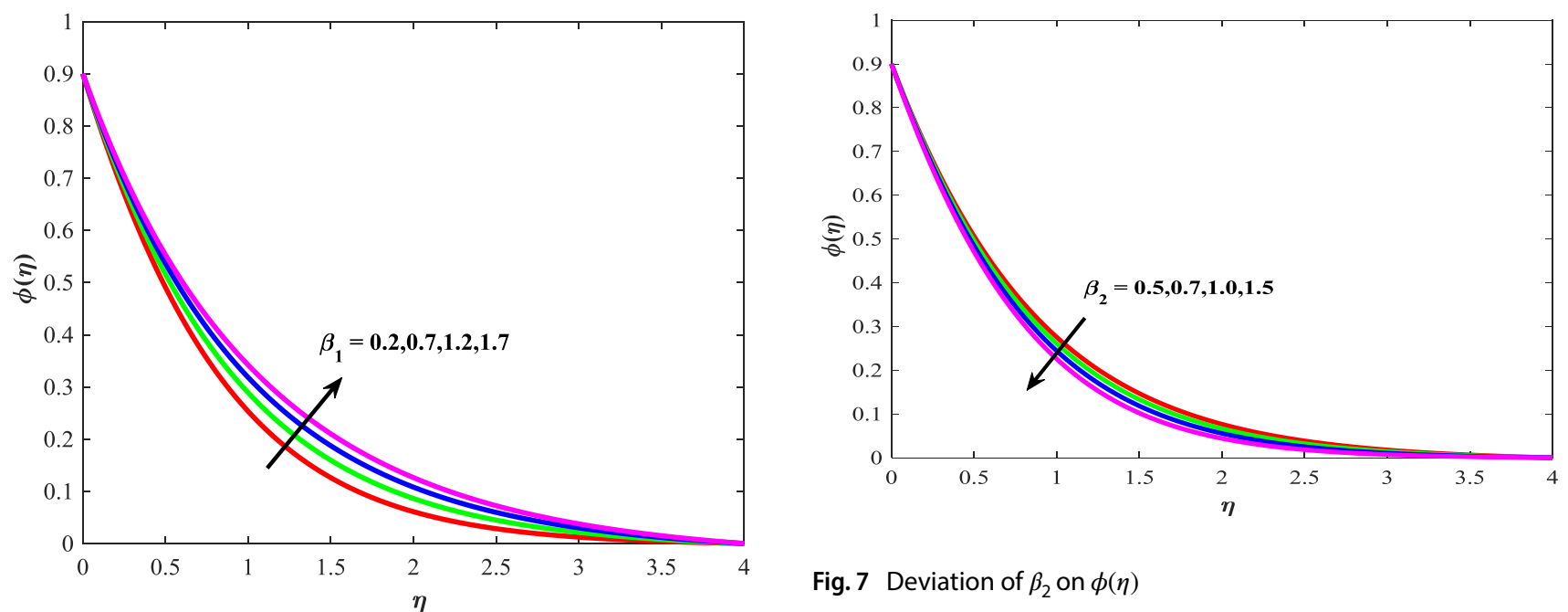

Fig. 7 Deviation of $\beta_{2}$ on $\phi(\eta)$

Fig. 4 Deviation of $\beta_{1}$ on $\phi(\eta)$
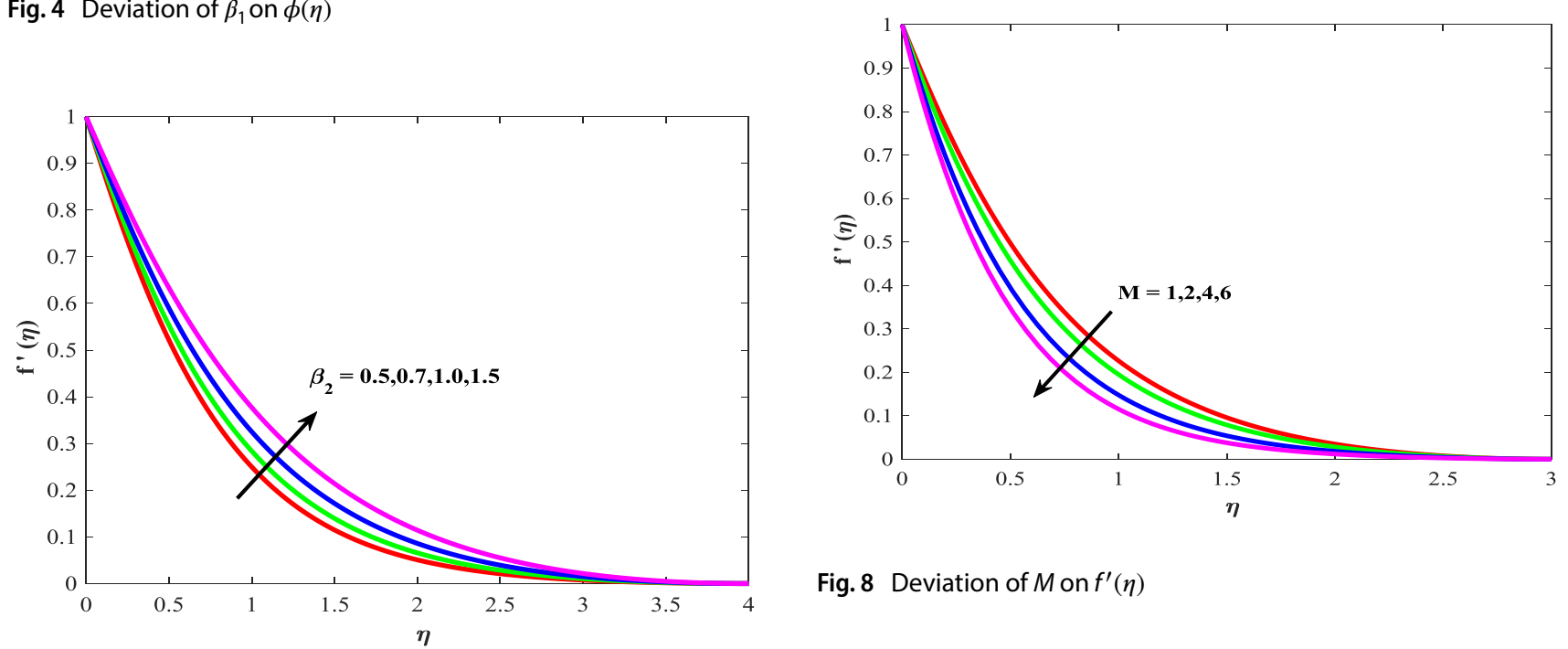

Fig. 8 Deviation of $M$ on $f^{\prime}(\eta)$

Fig. 5 Deviation of $\beta_{2}$ on $f^{\prime}(\eta)$
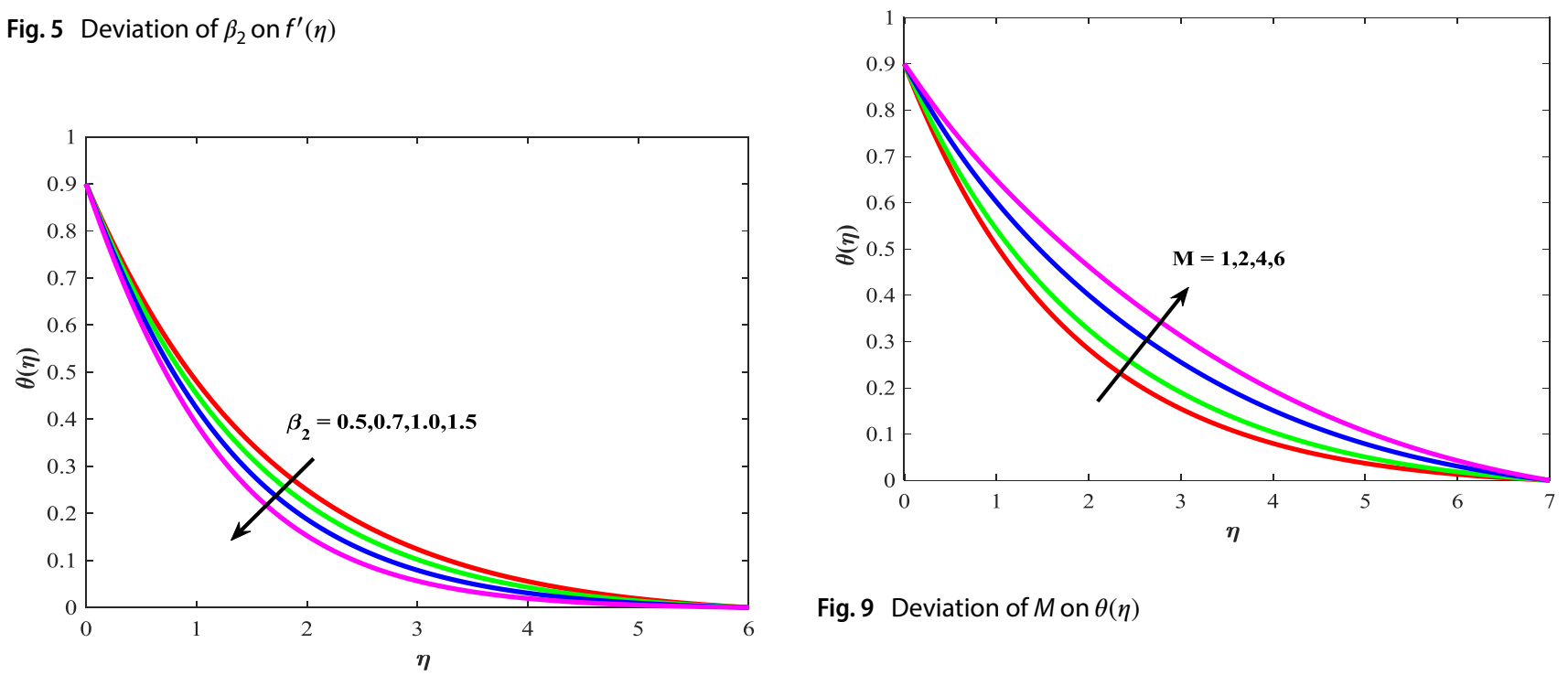

Fig. 9 Deviation of $M$ on $\theta(\eta)$

Fig. 6 Deviation of $\beta_{2}$ on $\theta(\eta)$ 


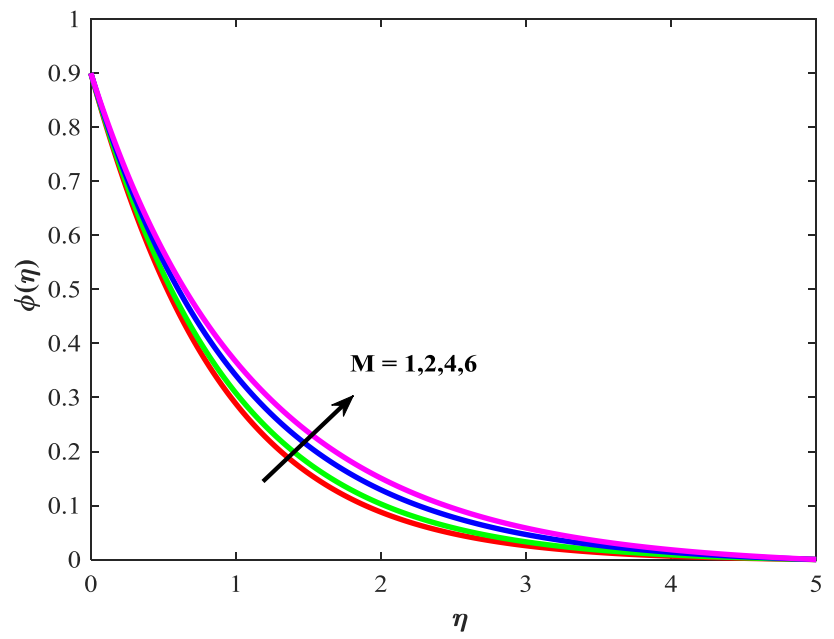

Fig. 10 Deviation of $M$ on $\phi(\eta)$

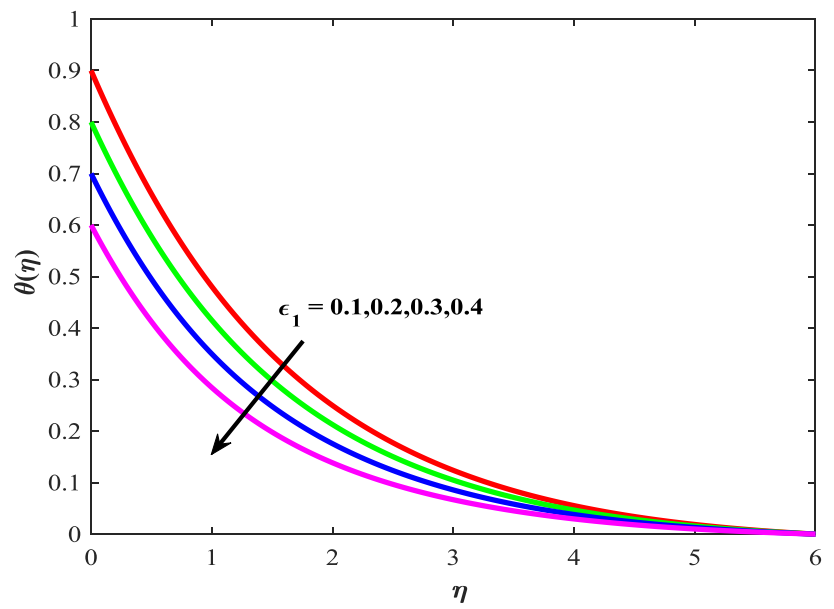

Fig. 11 Deviation of $\varepsilon_{1}$ on $\theta(\eta)$

that resists the flow. Consequently the motion of the fluid slows down leading to the depreciation of velocity. Figure 9 reveals that temperature is seen to increase with magnetic field, and hence, the corresponding thermal boundary layers are enlarged; since the fluid is decelerated, energy is dissipated in the form of heat leading to an increase in temperature in the thermal boundary layer. Figure 10 plots the solutal concentration response to magnetic field is qualitatively similar to that on temperature as illustrated in Fig. 9.

Figure 11 shows the effect of thermal stratification on temperature profile. Temperature gradient between boundary surface and ambient fluid decreases, leading to a reduction in temperature. Physically thermal stratification effect decreases the temperature difference between surface and the ambient fluid which leads to weaker temperature field. Figure 12 explores the influence of solutal

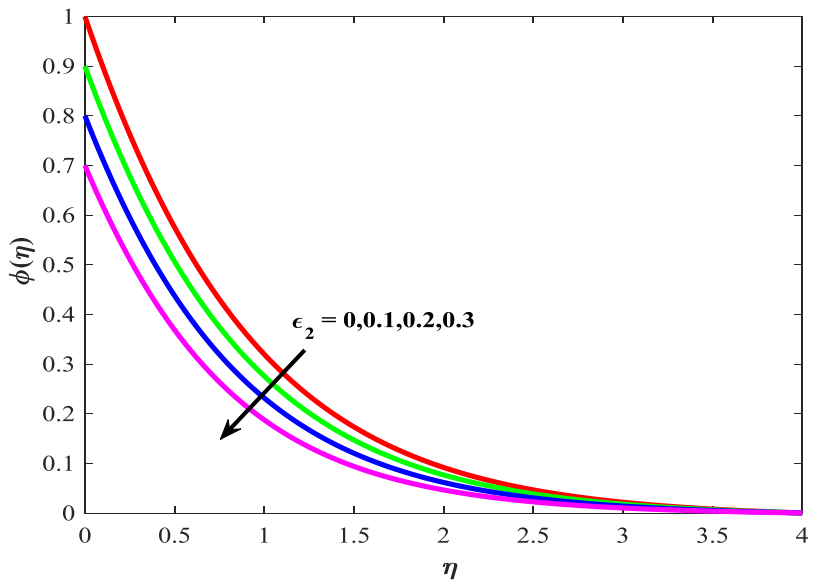

Fig. 12 Deviation of $\varepsilon_{2}$ on $\phi(\eta)$

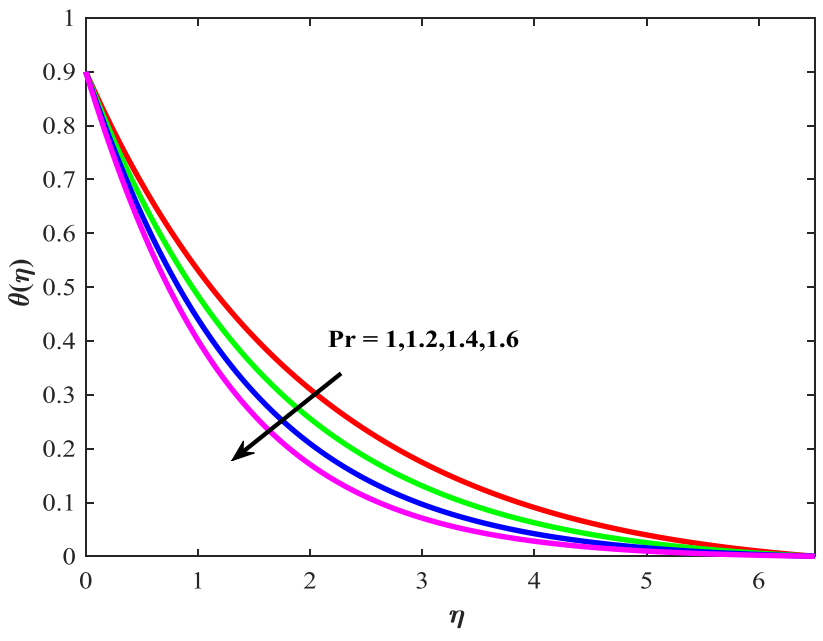

Fig. 13 Deviation of $\operatorname{Pr}$ on $\theta(\eta)$

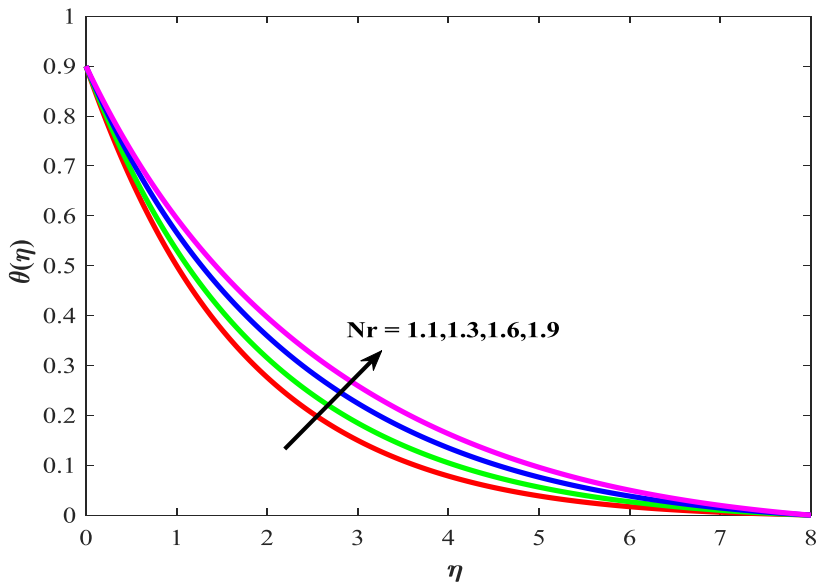

Fig. 14 Deviation of $\mathrm{Nr}$ on $\theta(\eta)$ 


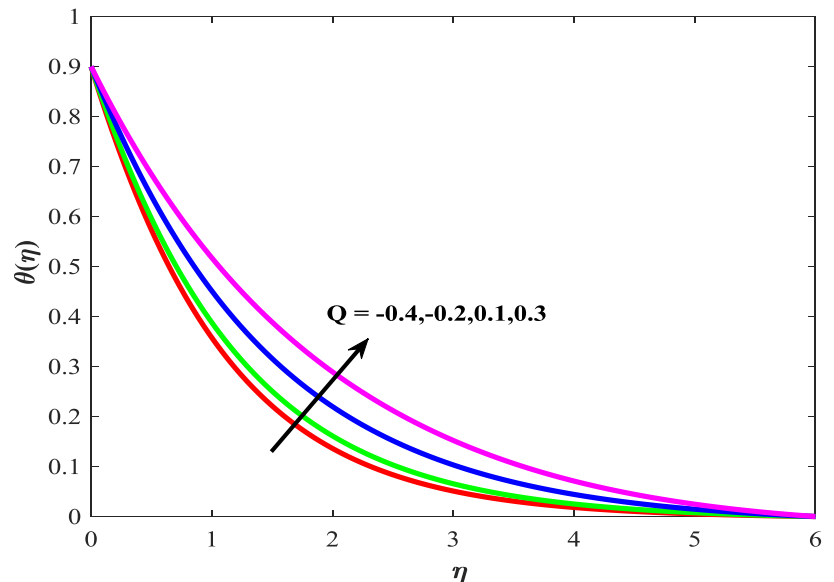

Fig. 15 Deviation of $Q$ on $\theta(\eta)$

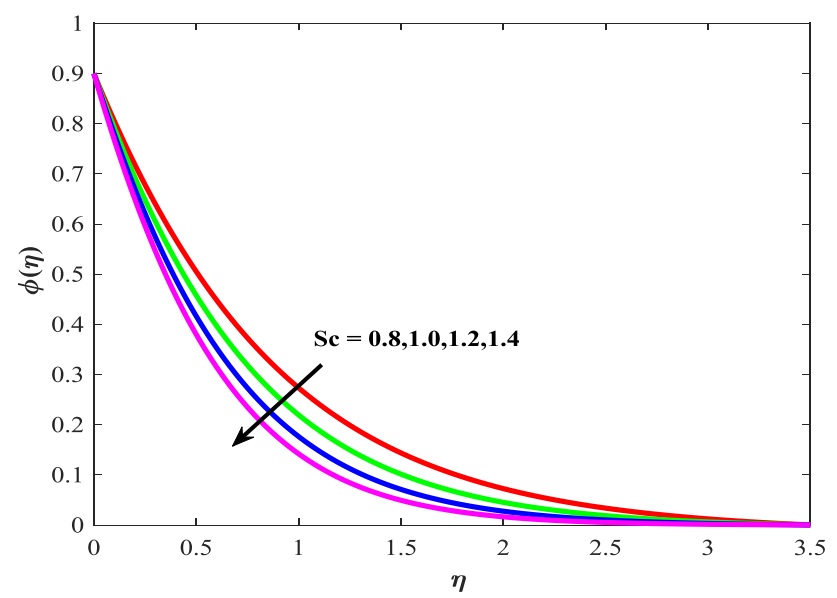

Fig. 16 Deviation of $S c$ on $\phi(\eta)$

stratification $\left(\varepsilon_{2}\right)$ on the concentration profile. It is clearly seen that species concentration falls significantly for increasing values of $\varepsilon_{2}$. Figure 13 is the plot of temperature showing the deviation of Prandtl number (Pr). It is seen that temperature diminishes when $P r$ is expanded as the fluid has low thermal diffusivity for higher Prandtl number which causes minimization in temperature. Figure 14 indicates the temperature profile for a variation in thermal radiation parameter $(\mathrm{Nr})$. Temperature and the thermal boundary layer thickness are enhanced with increasing values of $\mathrm{Nr}$. The fluid provides more heat for higher values of $\mathrm{Nr}$ which leads to enlarge the temperature and the thickness of thermal boundary layer.

Figure 15 presents the influence of heat source enhancing the temperature profile, since heat source discharges energy in the thermal boundary layer and augments the temperature. For increasing $Q>0$ (heat source), the temperature further increases. In the case of absorption of heat $Q<0$ (heat sink), temperature drops with decreasing

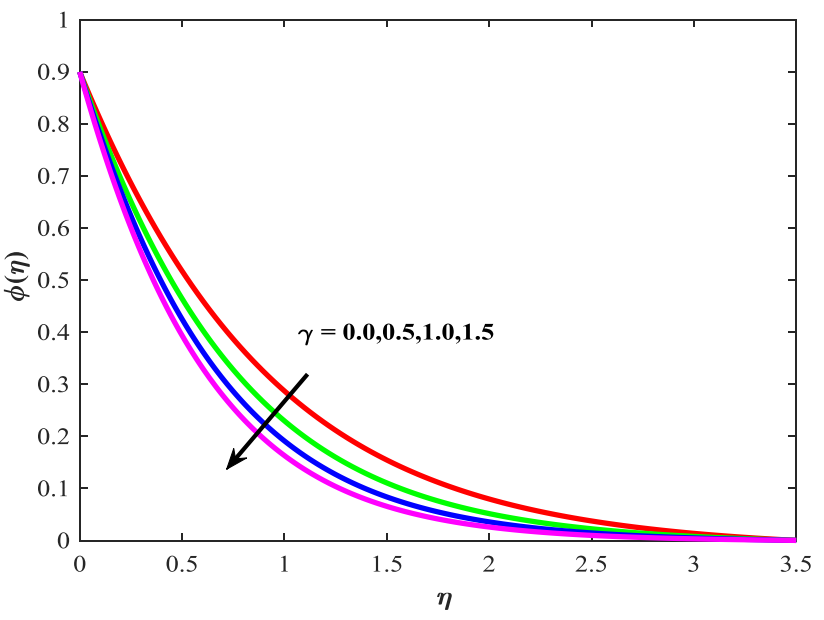

Fig. 17 Deviation of $\gamma$ on $\phi(\eta)$

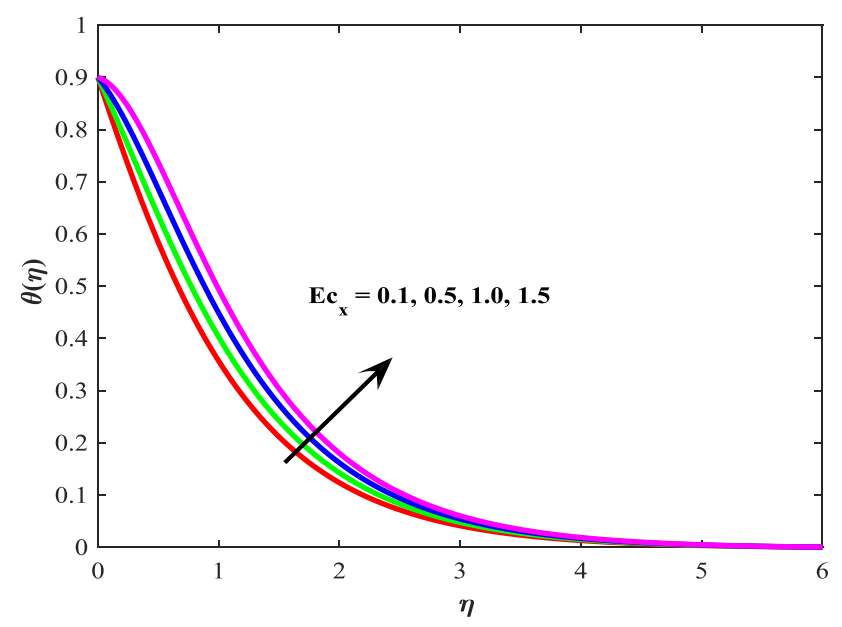

Fig. 18 Deviation of $E c_{x}$ on $\theta(\eta)$

values of $Q<0$ due to absorption of energy in the thermal boundary layer.

It is evident from Fig. 16 that the species concentration diminishes when Schmidt number increases owing to reduction in the solutal boundary layer thickness. It exhibits the larger diffusing species have high retarding influence on concentration profile. Figure 17 indicates that the non-dimensional concentration reduces for larger values of chemical reaction parameter $(\gamma)$. Higher chemical reaction parameter results in further reduction in the thickness of the solutal boundary layer. This results in watery molecular diffusivity and thinner boundary layers.

Figures 18 and 19 present that the temperature significantly increases for increasing values of $E c_{x}$ and $E c_{y}$. For $E c_{x}=E c_{y}=1.5$, we notice an overshoot of the temperature very close to the boundary. Consequently, the thickness of the thermal boundary layer increases in a region. 


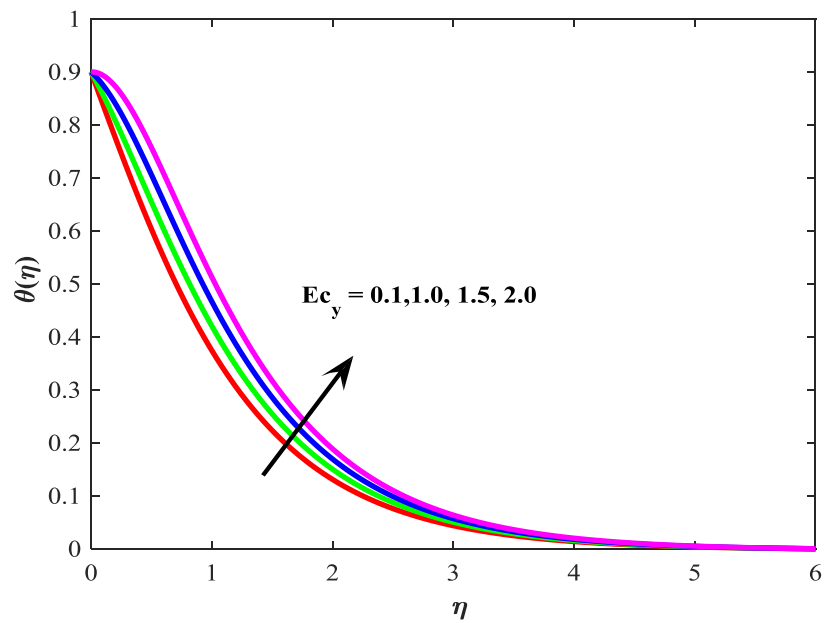

Fig. 19 Deviation of $E c_{y}$ on $\theta(\eta)$

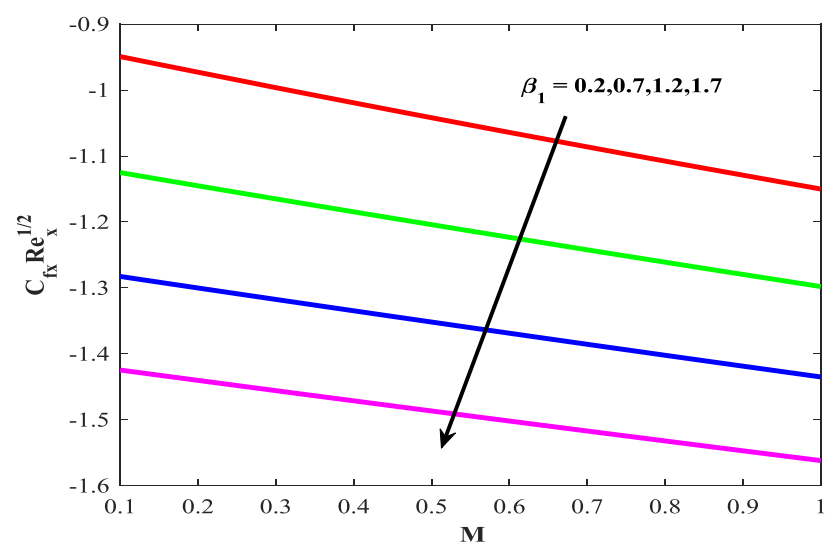

Fig. 20 Deviation of $M$ versus $\beta_{1}$ on $C_{f x}$

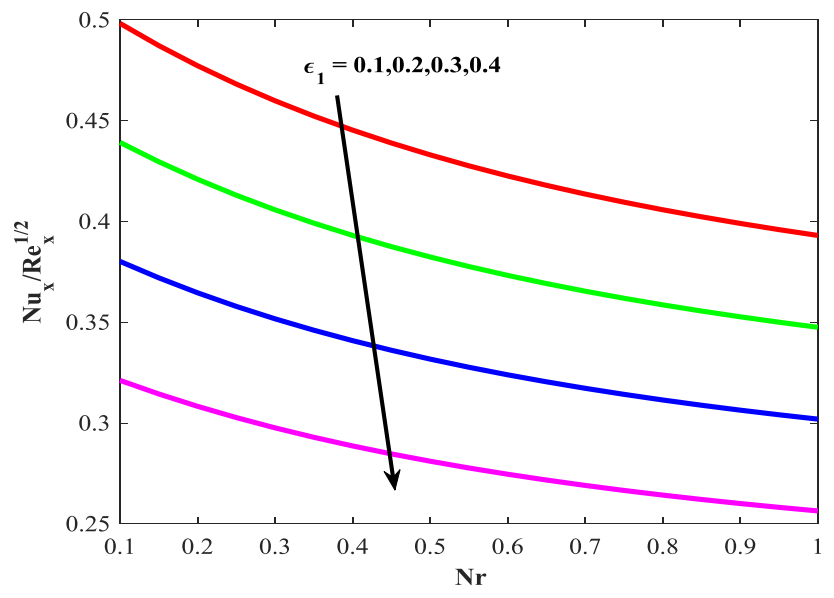

Fig. 21 Deviation of $N r$ versus $\varepsilon_{1}$ on $N_{u x}$

Physically, increase in Eckert number implies storage of heat energy in the fluid owing to the frictional heating. The coefficient of frictional drag is seen to decrease with

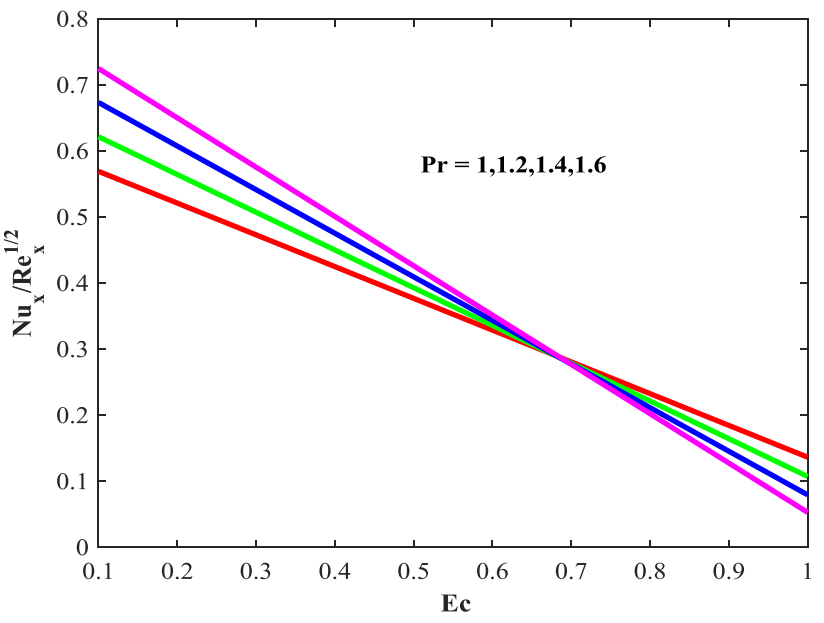

Fig. 22 Deviation of $E c_{x}$ versus $\operatorname{Pr}$ on $N_{u x}$

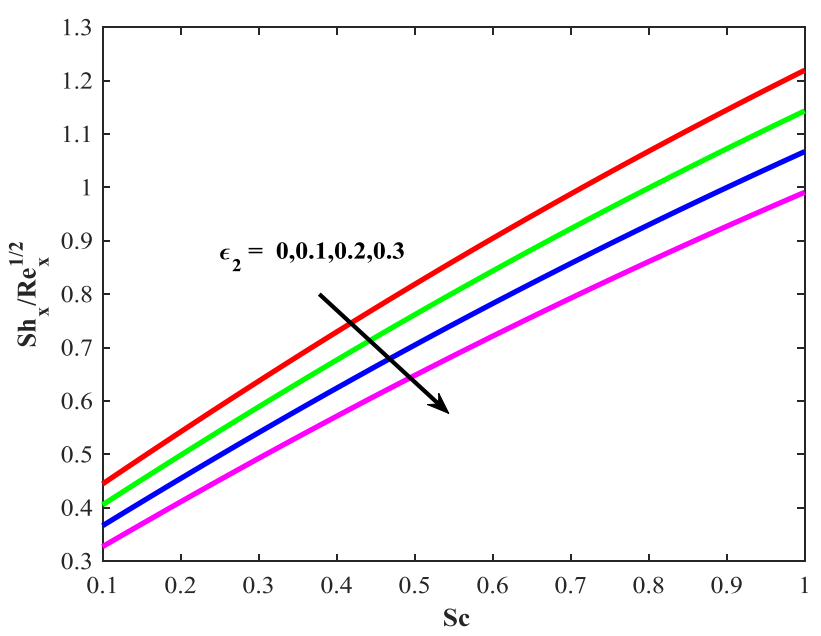

Fig. 23 Deviation of $S c$ versus $\varepsilon_{2}$ on $S h_{x}$

Deborah number $\left(\beta_{1}\right)$ and magnetic field parameters as plotted in Fig. 20. Nusselt number versus thermal radiation parameter varying thermal stratification parameter is drawn in Fig. 21. It is noticed that Nusselt number decreases significantly for increasing values of thermal radiation. Increasing values of thermal stratification parameter decrease Nusselt number uniformly. It exhibits from Fig. 22 that the Nusselt number is seen to enhance appreciably with Prandtl number while it depreciates with increasing Eckert number. It is noticed that when $E c_{x}>0.7$, the Nusselt number shows the reversal behavior as that of $E c_{x}<0.7$. Figure 23 projects the behavior of Sherwood number eliciting the influence of $\varepsilon_{2}$ and $\mathrm{Sc}$. The parameter $\varepsilon_{2}$ has a decreasing influence on Sherwood number while Schmidt number has a reversal effect. 


\section{Conclusions}

The concurrent effects of magnetic field and dual stratification of 3D Oldroyd-B fluid flow were analyzed in the direction of thermal radiation, viscous heating and heat generation or absorption. Some significant points of the problem are summarized below:

- The Deborah number $\left(\beta_{1}\right)$ and magnetic field parameters have a decreasing effect on velocity while temperature and concentration show a reverse behavior.

- The parameters $\varepsilon_{1}$ and Pr are reducing the temperature, whereas thermal radiation and heat source parameters have an increasing effect.

- Species concentration is depreciated with regard to Schmidt number, chemical reaction and solutal stratification parameters.

- Frictional drag coefficient is decreased with respect to the Deborah number $\left(\beta_{1}\right)$ and magnetic field parameters.

- Nusselt number increases with Prandtl number, while it decreases with parameters $\mathrm{Nr}$ and $\varepsilon_{1}$.

- Rate of mass transfer increases with solutal stratification parameter $\left(\varepsilon_{2}\right)$.

Authors' contributions All authors contributed equally to the writing of this paper. All authors read and approved the final manuscript.

\section{Compliance with ethical standards}

Conflict of interest The authors declare that they have no conflict of interest.

Human and animals rights This article does not contain any studies with human participants or animals performed by any of the authors.

\section{References}

1. Maxwell JC (1867) The dynamical theory of gases. Philos Trans R Soc Lond 157:49-88

2. Oldroyd JG (1950) The formulation of rheological equations of state. Proc R Soc Lond Ser 200(1063):523-541

3. Fetecau C (2003) A new exact solution for the flow of a Maxwell fluid past an infinite plate. Int J Non-Linear Mech 38:423-427

4. Huang C-J (2018) Effects of internal heat generation and Soret/ Dufour on natural convection of non-Newtonian fluids over a vertical permeable cone in a porous medium. J King Saud Univ Sci 30:106-111

5. Abdul Gaffar S, Ramachandra Prasad V, Keshava Reddy E (2017) Mixed convection boundary layer flows of a non-Newtonian Jeffrey's fluid from a non-isothermal wedge. Ain Shams Eng J 8:145-162

6. Ramana Reddy JV, Anantha Kumar K, Sugunammav SN (2017) Effect of cross diffusion on MHD non-Newtonian fluids flow past a stretching sheet with non-uniform heat source/sink: a comparative study. Alex Eng J 27:1829-1838

7. Raju CSK, Sandeep N, Gnaneswara Reddy M (2016) Effect of nonlinear thermal radiation on 3D Jeffrey fluid flow in the presence of homogeneous-heterogeneous reactions. Int J Eng Res Afr 21:52-68

8. Sandeep N, Gnaneswara Reddy M (2017) MHD Oldroyd-B fluid flow across a melting surface with cross diffusion and double stratification. Eur Phys J Plus 3:132-147

9. Hayat T, Sajid M (2007) Homotopy analysis of MHD boundary layer flow of an upper convected Maxwell fluid. Int J Eng Sci 45:393-401

10. Mukhopadhyay S, De Ranjan P, Layek GC (2013) Heat transfer characteristics for the Maxwell fluid flow past an unsteady stretching permeable surface embedded in a porous medium with thermal radiation. J Appl Mech Tech Phys 54(3):385-396

11. Hayat T, Abbas Z, Sajid M (2006) Series solution for the upper convected Maxwell fluid over a porous stretching plate. Phys Lett A 358:396-403

12. Ramzan M, Farooq M, Alhothuali MS, Malaikah HM, Cui W, Hayat T (2015) Three dimensional flow of an Oldroyd-B fluid with Newtonian heating. Int J Numer Methods Heat Fluid Flow 25(1):68-85

13. Sreelakshmi K, Sarojamma G, Makinde OD (2018) Dual stratification on the Darcy-Forchheimer flow of a Maxwell nanofluid over a stretching surface. Defect Diffus Forum 387:207-217

14. Kumar SG, Varma SVK, Prasad PD, Raju CSK, Makinde OD, Sharma $\mathrm{R}$ (2018) MHD reacting and radiating 3-D flow of Maxwell fluid past a stretching sheet with heat source/sink and soret effects in a porous medium. Defect Diffus Forum 387:145-156

15. Gireesha BJ, Archana M, Prasannakumara BC, Gorla R, Makinde OD (2017) MHD three dimensional double diffusive flow of Casson nano fluid with buoyancy forces and nonlinear thermal radiation over a stretching surface. Int J Numer Methods Heat Fluid Flow 27(12):2858-2878

16. Eswaramoorthi S, Bhuvaneswari M, Sivasankaran S, Makinde OD (2018) Heterogeneous and homogeneous reaction analysis on MHD Oldroyd-B fluid with Cattaneo-Christov heat flux model and convective heating. Defect Diffus Forum 387:194-206

17. Ibrahim SM, Kumar PV, Makinde OD (2018) Chemical reaction and radiation effects on non-Newtonian fluid flow over a stretching sheet with non-uniform thickness and heat source. Defect Diffus Forum 387:319-331

18. Khuzaimah Soid S, Ishak A, Pop I (2018) MHD flow and heat transfer over a radially stretching/shrinking disk. Chin J Phys 56(1):58-66

19. Hayat T, Nasir T, Khan MI, Alsaedi A (2018) Numerical investigation of MHD flow with Soret and Dufour effect. Results Phys 8:1017-1022

20. Siva Kumar Reddy B, Veera Krishna M, Surya Narayana Rao KV, BhuvanaVijaya R (2018) HAM solutions on MHD flow of nanofluid through saturated porous medium with Hall effects. Mater Today Proc 5(1):120-131

21. Muhammad T, Hayat T, Shehzad SA, Alsaedi A (2018) Viscous dissipation and Joule heating effects in MHD 3D flow with heat and mass fluxes. Results Phys 8:365-371

22. Nayak MK (2017) MHD 3D flow and heat transfer analysis of nano fluid by shrinking surface inspired by thermal radiation and viscous dissipation. Int J Mech Sci 124(125):185-193

23. Ganesh Kumar K, Ramesh GK, Gireesha BJ, Gorla RSR (2017) Characteristics of Joule heating and viscous dissipation on three-dimensional flow of Oldroyd-B nano fluid with thermal radiation. Alex Eng J 3:2139-2149

24. Muhammad T, Hayat T, Shehzad SA, Alsaedi A (2018) Viscous dissipation and Joule heating effects in MHD 3D flow with heat and mass fluxes. Results Phys 8:365-371 
25. Jaber KK (2016) Joule heating and viscous dissipation on effects on MHD flow over a stretching porous sheet subjected to power law heat flux in presence of heat source. Open J Fluid Dyn 6:156

26. Qayyum S, Hayat T, Shehzad SA, Alsaedi A (2018) Mixed convection and heat generation/absorption aspects in MHD flow of tangent-hyperbolic nano liquid with Newtonian heat/mass transfer. Radiat Phys Chem 144:396-404

27. Mishra SR, Bhatti MM (2017) Simultaneous effects of chemical reaction and Ohmic heating with heat and mass transfer over a stretching surface: a numerical study. Chin J Chem Eng 25:1137-1142

28. Khan KA, Butt AR, Raza N (2018) Effects of heat and mass transfer on unsteady boundary layer flow of a chemical reacting Casson fluid. Results Phys 8:610-620

29. Rehman KU, Malik MY, Salahuddin T, Naseer M (2016) Dual stratified mixed convection flow of Eyring-Powell fluid over an inclined stretching cylinder with heat generation/absorption effect. AIP Adv 6:075112

30. Rehman KU, Malik AA, Malik MY, Sandeep N, Saba NU (2017) Numerical study of double stratification in Casson fluid flow in the presence of mixed convection and chemical reaction. Results Phys 7:2997-3006

31. Harris J (1977) Rheology and non-Newtonian flow. Longman, London

32. Hayat T, Muhammad T, Shehzad SA, Alsaedi A (2015) Temperature and concentration stratification effects in mixed convection flow of an Oldroyd-B fluid with thermal radiation and chemical reaction. PLOS One 10:1-23
33. Gireesha BJ, Ramesh GK, Ganesh Kumar K, Prasannakumar BC (2018) Nonlinear convective heat and mass transfer of Oldroyd$B$ nano fluid over a stretching sheet in the presence of uniform heat source/sink. Results Phys 9:1555-1563

34. Ibrahim W, Shankar B (2013) MHD boundary layer flow heat transfer of a nano fluid past a permeable stretching sheet with velocity thermal and solutal slip boundary conditions. Comput Fluids 75:1-10

35. Hayat T, Imtiaz M, Alsaedi A (2016) Unsteady flow of nano fluid with double stratification and magnetohydrodynamics. Int J Heat Mass Transf 92:100-109

36. Hayat T, Shehzad SA, Alsaedi A (2013) Three-dimensional stretched flow of Jeffrey fluid with variable thermal conductivity and thermal radiation. Appl Math Mech 34:823-832

37. Rudraswamy NG, Shehzad SA, Ganesh Kumar K, Gireesha BJ (2017) Numerical analysis of MHD three-dimensional Carreau nanoliquid flow over bidirectionally moving surface. J Braz Soc Mech Sci Eng 39:5037-5047

38. Ganesh Kumar K, Rudraswamy NG, Gireesha BJ, Krishnamurthy MR (2017) Influence of nonlinear thermal radiation and viscous dissipation on three dimensional flow of Jeffrey nano fluid over a stretching sheet in the presence of Joule heating. Nonlinear Eng 6(3):207-219

Publisher's Note Springer Nature remains neutral with regard to jurisdictional claims in published maps and institutional affiliations. 